\title{
On non-additive processes
}

\author{
ULRICH WACKER \\ Fakultät für Mathematik und Informatik der Universität Passau, Innstr. 27, \\ D-8390 Passau, West Germany
}

(Received 16 April 1984)

Abstract. The aim of this paper is to introduce and study the class of boundedly non-additive processes. The main result is the decomposition in theorem (2.1) and theorem (3.1), which says that a boundedly non-additive process is the sum of a non-positive subadditive, a non-negative superadditive and an additive process. By this decomposition we can extend the mean and local ergodic theorems for superadditive processes of $\mathrm{M}$. A. Akcoglu and U. Krengel to boundedly non-additive processes. At the end of this paper some examples are given.

\section{Definitions}

For $a=\left(a_{i}\right), b=\left(b_{i}\right) \in \mathbb{R}^{\prime}, l \geq 1$ we set:

$a \leq b(a<b): \Leftrightarrow a_{i} \leq b_{i},\left(a_{i}<b_{i}\right)$, for $1 \leq i \leq l$;

$\left[a, b\left[:=\left\{u \in \mathbb{R}^{l}: a \leq u<b\right\}, \quad \prod\left(\left[a, b[):=\prod_{i=1}^{l}\left(b_{i}-a_{i}\right)\right.\right.\right.\right.$;

$0=(0,0, \ldots, 0), \quad e=(1,1, \ldots, 1) ; \mathbb{R}_{+}^{\prime}:=\left\{a \in \mathbb{R}^{\prime}: a \geq 0\right\}$;

$I_{d}:=\left\{\left[a, b\left[: a, b \in \mathbb{N}_{0}^{I}\right\}\right.\right.$, with $\mathbb{N}_{0}:=\{0,1,2, \ldots\} ;$ and finally

$I_{s}:=\left\{\left[a, b\left[: a, b \in \mathbb{R}_{+}^{l}\right\}\right.\right.$.

Let $\tau=\left(\tau_{u}\right)_{u \in I^{\prime}}\left(I=\mathbb{N}_{0}^{l}\right.$ or $\left.\mathbb{R}_{+}^{l}\right)$ be a measurable semigroup of measure preserving transformations on a measure space $(X, Q, P)$. For a $Q$-measurable function $f$ we denote the equivalence class of all functions, which are a.s. equal to $f$, by $\bar{f}$. Let $\mathcal{M}$ be the vector lattice of these equivalence classes, and let $T=\left(T_{u}\right)_{u \in I}$ be the semigroup of linear operators acting on $\mathcal{M}$ by the relation $T_{u} \bar{f}=\bar{f}\left(\tau_{u} \cdot\right)$.

Let $F$ be a set function defined on $I_{d}\left(I_{s}\right)$ with values in $\mathcal{M}$. We distinguish the following conditions:

(1.1) $T_{u} F_{J}=F_{J+u} \quad$ whenever $J \in I_{d}\left(I_{s}\right)$ and $u \in \mathbb{N}_{0}^{l}\left(\mathbb{R}_{+}^{l}\right)$;

(1.2) if $J_{1}, \ldots, J_{n}$ are disjoint sets in $I_{d}\left(I_{s}\right)$ and if $J=\bigcup_{i=1}^{n} J_{i}$ is also in $I_{d}\left(I_{s}\right)$ then $F_{J} \geq \sum_{i=1}^{n} F_{J_{i}}$;

(1.3) $\sup \left\{\Pi(J)^{-1} \int F_{J} d P: J \in I_{d}\left(I_{s}\right), \Pi(J)>0\right\}=\gamma=\gamma(F)<+\infty$,

(1.4) $\int F_{[0, e[}^{-} d P<+\infty$, where $f^{+}, f^{-}$denote the positive and negative part of an $f \in \mathcal{M}$.

Definition (1.5). If $F$ satisfies (1.1), we will say that $F$ is a stationary process with discrete (or continuous) parameter. If $F$ also satisfies (1.2), (1.3), and takes values in $L_{1}, F$ is called a superadditive process. If $-F$ is a superadditive process, $F$ is called a subadditive process. $F$ is called an extended superadditive process if $F$ satisfies $(1.1),(1.2)$ and (1.4). If $-F$ is an extended superadditive process, $F$ is 
called an extended subadditive process. $F$ is called an additive process, if it is a superadditive and a subadditive process.

For the next definition we need some further notation. We denote a set by the letter $M$, iff it is contained in $I_{d}\left(I_{s}\right)$ and its elements are disjoint sets. For two sets $M_{1}, M_{2}$ we write $M_{1} \triangleleft M_{2}$, if every element in $M_{1}$ is the disjoint union of elements of $M_{2}$. For $I \in I_{d}$ we set

$$
M_{I}:=\left\{\left[u, u+e\left[: u \in I \cap \mathbb{N}_{0}^{\prime}\right\}\right.\right.
$$

For $I \in I_{d}\left(I_{s}\right)$ and $\{I\} \triangleleft M_{1} \triangleleft M_{2}$, we define

$$
\begin{aligned}
& d^{\prime \prime}\left(M_{1}, M_{2}\right):=\sum_{I \in M_{1}}\left|F_{I}-\sum_{\substack{J \in M_{2} \\
J \in I}} F_{J}\right| \\
& d^{*}\left(M_{1}, M_{2}\right):=\sum_{I \in M_{1}}\left(F_{I}-\sum_{\substack{J \in M_{2} \\
J \subset I}} F_{J}\right) \dot{ } \quad \text { for } *=+,-.
\end{aligned}
$$

For $A=\left(M_{i}\right)_{1 \leq i \leq m}, m \in \mathbb{N}$, with $\{I\}=M_{1} \triangleleft M_{2} \triangleleft \cdots \triangleleft M_{m}$, where $M_{m}$ consists only of finitely many elements, we define:

$$
F_{I}^{*}(A):=\sum_{i=1}^{m-1} d^{*}\left(M_{i}, M_{i+1}\right), \quad *=",+,-
$$

Let $\bar{A}(I)$ be the set of all such $A$. In the discrete parameter case we define

$$
F_{I}^{*}:=\max \left\{F_{I}^{*}(A): A \in \bar{A}(I)\right\}, \quad *={ }^{\prime \prime},+,-,
$$

and

$$
p(F):=\sup \left\{\Pi(I)^{-1} \int F_{I}^{\prime \prime} d P: \Pi(I)>0, I \in I_{d}\right\}
$$

In the continuous parameter case we suppose that for every countable $K \subset \bar{A}(I)$, $\sup \left\{F_{I}^{*}(A): A \in K\right\}, *=",+,-$, is in $L_{1}$ and their integrals are uniformly bounded. Then $F_{I}^{*}:=\sup \left\{F_{I}^{*}(A): A \in \bar{A}(I)\right\}, *=",+,-$, exists and is in $L_{1}$. If this is satisfied for every $I \in I_{s}$ we define:

$$
p(F):=\sup \left\{\Pi(I)^{-1} \int F_{I}^{\prime \prime} d P: \Pi(I)>0, I \in I_{s}\right\} .
$$

Definition (1.6). A stationary process $F$ is called locally boundedly non-additive if $F_{I}^{\prime \prime}$ is integrable for some $I$ with $\Pi(I)>0$. It is called boundedly non-additive if $p(F)$ is finite.

$p(\cdot)$ is a seminorm on the vector space of all stationary, boundedly non-additive processes, with a common semigroup $T$. If we build the canonical quotient space relative to $p$, we obtain a Banach space, where two processes are identified iff their 
difference is additive. This can be seen easily. The only non-trivial part is the completeness of the quotient space. Let $\left\{F_{n}^{\prime}\right\}_{n \in N}$ be a Cauchy sequence in the quotient space, and $F_{n}$ a representative of $F_{n}^{\prime}$. If $\left\{n_{i}\right\}$ increases fast enough we have

$$
p\left(F_{n_{i}}-F_{n_{i-1}}\right)<2^{-i}, \quad i \geq 2 .
$$

Then the processes $F^{*}, *=+$, - defined by

$$
F_{I}^{*}=\sum_{i=2}^{\infty}\left(F_{n_{i}, I}-F_{n_{i-1}, I}\right)^{*}+F_{n_{1}, I}^{*}, \quad *=+,-; \quad I \in I_{d}\left(I_{s}\right),
$$

exist and they are non-negative superadditive processes. The equivalence class of $F$, which is defined by

$$
F_{I}=F_{I}^{+}-F_{I}^{-}, \quad I \in I_{d}\left(I_{s}\right),
$$

is the limit point of the sequence. Let $F$ be a superadditive process, then we obtain the following relationship between the constants $\gamma(F)$ and $p(F)$.

In the discrete parameter case:

$$
p(F)=\gamma(F)-\int F_{[0, e[} d P .
$$

And in the continuous parameter case, if in addition $\sup \left\{\Pi(I)^{-1} \int\left|F_{I}\right| d P: I \in I_{s}\right.$, $\Pi(I)>0\}$ is finite:

$$
p(F)=\gamma(F)-s_{F}
$$

where $s_{F}$ is defined by

$$
s_{F}:=\lim _{i \rightarrow 0+} \prod\left(\left[0, t \cdot e[)^{-1} \int F_{[0, t e[} d P .\right.\right.
$$

For the second equality see the proof of lemma (4.7) in [1].

2. The discrete parameter case

ThEOREM (2.1). Let $F$ be a stationary process with discrete parameter. Then

$$
F=F^{+}-F^{-}+G \text {, }
$$

where $G$ is defined by

$$
G_{I}:=\sum_{u \in \operatorname{InN_{0}^{\prime }}} F_{[u, u+e[}
$$

Furthermore, $F^{+}$and $F^{-}$are non-negative extended superadditive processes. If $p(F)$ is finite, then $F^{+}$and $F^{-}$are superadditive processes.

Proof. For $A \in \bar{A}(I)$ with $A=\left(M_{i}\right)_{1 \leq i \leq m}$ and $M_{m} \neq M_{I}$ we obtain

$$
F_{I}^{*}(A) \leq F_{I}^{*}\left(A^{\prime}\right), \quad *=",+,-,
$$

with $A^{\prime}=\left(M_{1}, \ldots, M_{m}, M_{l}\right)$. Let $A^{*}(I)$ be the set of all $A \in \bar{A}(I)$ of the form $A=\left(M_{1}, \ldots, M_{m}, M_{I}\right)$. We obtain:

$$
F_{I}^{*}=\sup \left\{F_{I}^{*}(A): A \in A^{*}(I)\right\}, \quad *=",+,- \text {. }
$$


So $\bar{A}(I)$ can be replaced by $A^{*}(I)$. First we show (2.1.1) and

$$
F_{I}^{+}+F_{I}^{-}=F_{I}^{\prime \prime} \quad \text { for every } I \in I_{d}
$$

For every $A \in A^{*}(I)$ we obtain

$$
F_{I}^{+}(A)-F_{I}^{-}(A)=F_{I}-\sum_{J \in M_{l}} F_{J}
$$

Let $A_{i} \in A^{*}(I), 1 \leq i \leq N$. The last equality implies

$$
\begin{aligned}
F_{I}^{+}\left(A_{1}\right) \vee \cdots \vee F_{I}^{+}\left(A_{N}\right)= & \left(\left(F_{I}-\sum_{J \in M_{I}} F_{J}\right)+F_{I}^{-}\left(A_{1}\right)\right) \\
& \vee \cdots \vee\left(\left(F_{I}-\sum_{J \in M_{I}} F_{J}\right)+F_{I}^{-}\left(A_{N}\right)\right) \\
= & \left(F_{I}-\sum_{J \in M_{I}} F_{J}\right)+F_{I}^{-}\left(A_{1}\right) \vee \cdots \vee F_{I}^{-}\left(A_{N}\right) .
\end{aligned}
$$

Hence

$$
F_{I}^{+}\left(A_{1}\right) \vee \cdots \vee F_{I}^{+}\left(A_{N}\right)-F_{I}^{-}\left(A_{1}\right) \vee \cdots \vee F_{I}^{-}\left(A_{N}\right)=F_{I}-\sum_{J \in M_{I}} F_{J}
$$

In the case $\left\{A_{1}, \ldots, A_{N}\right\}=A^{*}(I)$ we obtain (2.1.1). We further obtain

$$
\begin{aligned}
F_{I}^{+}+F_{I}^{-} & =2 F_{I}^{-}+\left(F_{I}-\sum_{J \in M_{I}} F_{J}\right) \\
& =\left(2 F_{I}^{-}\left(A_{1}\right)+\left(F_{I}-\sum_{J \in M_{I}} F_{J}\right)\right) \vee \cdots \vee\left(2 F_{I}^{-}\left(A_{N}\right)+\left(F_{I}-\sum_{J \in M_{I}} F_{J}\right)\right) \\
& =F_{I}^{\prime \prime}\left(A_{1}\right) \vee \cdots \vee F_{I}^{\prime \prime}\left(A_{N}\right)=F_{I}^{\prime \prime} .
\end{aligned}
$$

By definition $F^{+}, F^{-}$, and $G$ satisfy $(1.1)$, and we have $F^{*} \geq 0$ for $*=",+,-$. Next we shall prove (1.2) for $F^{+}$. For $F^{-}$the proof is the same.

Let $I_{j}, 1 \leq j \leq n$, in $I_{d}$ be disjont sets, such that $I=\bigcup_{j=1}^{n} I_{j}$ is also in $I_{d}$. Take

$$
A_{j}^{p}:=\left(M_{1, j}^{p}, \ldots, M_{l_{j}, j}^{p}\right) \in A^{*}\left(I_{j}\right), \quad 1 \leq p \leq N_{j},
$$

such that $F_{I_{j}}^{+}=F_{I_{i}}^{+}\left(A_{j}^{1}\right) \vee \cdots \vee F_{I_{j}}^{+}\left(A_{j}^{N_{j}}\right)$. Put $l=\max l_{j, p}$. For $\bar{p}=\left(p_{1}, \ldots, p_{n}\right), 1 \leq p_{j} \leq$ $N_{j}$, let $M_{1}^{\bar{p}}=\{I\}$, and for $1 \leq k \leq l$ let $M_{k+1}^{\bar{p}}$ be the collection of all sets in $\bar{M}_{k_{j}}^{p_{j}}$, $1 \leq j \leq n$, where we set $\bar{M}_{k, j}^{p_{j}}=M_{k, j}^{p_{j}}$; if $k \leq l_{j, p_{j}}$ and $\bar{M}_{k, j}^{p_{j}}=M_{I_{j}}$ otherwise. Set $A^{\bar{p}}=$ $\left(M_{1}^{\bar{p}}, \ldots, M_{l+1}^{\bar{P}_{1}}\right)$, then we obtain

$$
F_{I}^{+} \geq \max _{e \leq \bar{p} \leq \bar{N}} F_{I}^{+}\left(A^{\bar{p}}\right) \geq \sum_{j=1}^{n} F_{I_{j}}^{+}
$$

with $\bar{N}=\left(N_{1}, \ldots, N_{n}\right)$, and (1.2) is proved.

(2.1.3) implies $\gamma\left(F^{+}\right)+\gamma\left(F^{-}\right)=p(F)$, and this proves the last statement in theorem (2.1).

The decomposition (2.1.1) is minimal in the sense that for every decomposition $F=H^{1}-H^{2}+H$, with non-negative superadditive processes $H_{1}, H_{2}$ and additive $H, F_{I}^{+} \leq H_{I}^{1}$ and $F_{I}^{-} \leq H_{I}^{2}$, for $I \in I_{d}$, is satisfied. For equivalence classes $\bmod P$, $\lim _{n \rightarrow \infty} f_{n}=f$ a.s. means that $\lim _{n \rightarrow \infty} \tilde{f}_{n}=\tilde{f}$ a.s. is satisfied if $\tilde{f}_{n}, \tilde{f}$ are representatives of $f_{n}, f$. Theorem (2.1) and theorem (2.5) in [1] yield: 
THEOREM (2.2). Let $F$ be a stationary process with discrete parameter, which satisfies $F_{[0, e[} \in L_{1}$ and $p(F)<+\infty$. Set $J_{n}:=[0, n \cdot e[, n \in \mathbb{N}$. Then

$$
\lim _{n \rightarrow \infty} \prod\left(J_{n}\right)^{-1} F_{J_{n}}
$$

exists a.s.

The theorem remains valid if $\left(J_{n}\right)_{n \in \mathbb{N}}$ is a regular family of sets with $\lim _{n \rightarrow \infty} J_{n}=\mathbb{P}_{0}^{l}$, as defined in [1]. If $(X, Q, P)$ is a finite measure space, then we can replace the condition $p(F)<+\infty$ by the existence of the time constant of one of the processes $F^{+}$and $F^{-}$in (2.1.1). E.g. we can suppose $\gamma\left(F^{-}\right)<+\infty$. Then, by theorem (2.5) in [1], $\lim _{n \rightarrow \infty} \prod\left(J_{n}\right)^{-1} F_{J_{n}}^{-}$and $\lim _{n \rightarrow \infty} \Pi\left(J_{n}\right)^{-1} G_{J_{n}}$ exist a.s. and are in $L_{1}$. By a truncation argument like that in $\left[4\right.$, p.188] the existence of $\lim _{n \rightarrow \infty} \prod\left(J_{n}\right)^{-1} F_{J_{n}}^{+}$follows from theorem (2.5) in [1]. Together these prove the last statement. Let $F$ be a stationary process with discrete parameter on a probability space $(X, Q, P)$ with $F_{[n, k]} \in L_{1}$ for $\left[n, k\left[\in I_{d}\right.\right.$. Y. Derriennic [2] proved that $(1 / N) F_{[0, N[}$ converges a.s. and in $L_{1}$, if the following two conditions are satisfied:

(a) there is a sequence $\left(h_{k}\right)_{k \in \mathbb{N}} \subset L_{1}, h_{k} \geq 0$, with $\sup _{k \geq 1}\left\|h_{k}\right\|_{1}<+\infty$ and

$$
F_{[0, n+k[}-F_{[0, n[}-F_{[n, n+k[} \leq T^{n} h_{k} \quad\left(T=T_{1}\right),
$$

for every $n$ and $k$,

$$
\text { (b) } \inf _{N \geq 1}(1 / N) \int F_{[0, N[} d P>-\infty \text {. }
$$

From this result the Shannon-McMillan-Breiman theorem follows at once (see [2]).

If we replace $\sup _{k \geq 1}\left\|h_{k}\right\|_{1}<+\infty$ by $\sup _{k \geq 1} h_{k} \in L_{1}$ in (a), then $F$ is boundedly non-additive. The question arises as to whether all processes which satisfy (a) and (b) are boundedly non-additive.

\section{The continuous parameter case}

THEOREM (3.1). Let $F$ be a stationary process with continuous parameter which satifies $p(F)<+\infty$. Then

$$
F=F^{+}-F^{-}+G
$$

where $F^{+}$and $F^{-}$are non-negative superadditive processes and $G$ is additive.

Proof. Fix $I \in I_{s}$. As $F$ is boundedly non-additive, there is a countable set $K=$ $\left\{A_{1}, \ldots\right\} \subset \bar{A}(I)$ with

$$
F_{I}^{*}=\sup \left\{F_{I}^{*}(A): A \in K\right\}, \quad *=",+,-.
$$

Put $f_{N}^{*}:=F_{I}^{*}\left(A_{1}\right) \vee \cdots \vee F_{I}^{*}\left(A_{N}\right)$. We obtain $f_{N}^{*} \uparrow F_{I}^{*}$ and $f_{N}^{\prime \prime}=f_{N}^{+}+f_{N}^{-}$. Hence

$$
F_{I}^{\prime \prime}=F_{I}^{+}+F_{I}^{-} \text {, }
$$

Put $g_{N}:=F_{I}-f_{N}^{+}+f_{N}$, and let $G$ be defined by $G_{I}:=\lim _{N \rightarrow \infty} g_{N}$. Now we will show, that $F^{*}, G$ and $-G$ satisfy (1.2). Together with (3.1.2) this implies that the $F^{*}$ are superadditive processes. Let $I_{1}, \ldots, I_{m}$ be disjoint sets in $I_{s}$ such that $\bigcup_{i=1}^{m} I_{i}=I$. Take $K_{i}=\left\{A_{1}^{i}, A_{2}^{i}, \ldots\right\} \subset \bar{A}\left(I_{i}\right)$ with

$$
F_{I}^{*}=\sup \left\{F_{I}^{*}(A): A \in K_{i}\right\}, \quad 1 \leq i \leq m .
$$


Put $f_{N}^{* i}=F_{I_{i}}^{*}\left(A_{1}^{i}\right) \vee \cdots \vee F_{I_{i}}^{*}\left(A_{N}^{i}\right)$. By the same argument as in the proof of theorem (2.1), we obtain $B_{1}^{N}, \ldots B_{M}^{N} \in \bar{A}(I)$ with

$$
F_{I}^{*}\left(B_{1}^{N}\right) \vee \cdots \vee F_{I}^{*}\left(B_{M}^{N}\right) \geq \sum_{i=1}^{m} f_{N}^{*, i}
$$

So for every $N \in \mathbb{N}, F_{I}^{*} \geq \sum_{i=1}^{m} f_{N}^{*, i}$ is satisfied, and (1.2) is proved for $F^{*}, *=",+,-$. It remains to prove

$$
G_{I}=\sum_{i=1}^{m} G_{I_{i}}
$$

Let $A_{j}=\left(M_{1}^{j}, \ldots, M_{K_{j}}^{j}\right)$ and $A_{j}^{i}=\left(M_{1, i}^{j}, \ldots, M_{K_{j, i},}^{j}\right), 1 \leq i \leq m, j \in \mathbb{N}$. For $1 \leq i \leq m$ let $M_{i}^{N}$ be the collection of all intersections of sets in $M_{K_{j, i}, i}^{j}$ and in $M_{K_{j}, 1 \leq j \leq N}^{j}$. Let $M_{N}$ be the collection of all these intersections. We set

$$
Z_{N}^{i}=\sum_{J \in M_{i}^{N}} F_{J} \text { and } Z_{N}=\sum_{J \in M_{N}} F_{J}
$$

Because $\sum_{i=1}^{m} Z_{N}^{i}=Z_{N}$ is satisfied, (3.1.3) follows from

$$
Z_{N}^{i} \rightarrow G_{I_{i}} \text { and } Z_{N} \rightarrow G_{I}
$$

By $M_{i}^{N} \triangleleft M_{K_{j, i}, i}^{j}$ and $M_{N} \triangleleft M_{K_{j}}^{j}$ for $1 \leq j \leq N$, we obtain

$$
A_{j, N}^{i}:=\left(M_{1, i}^{j}, \ldots, M_{K_{j, i}, i}^{j}, M_{i}^{N}\right) \in \bar{A}\left(I_{i}\right)
$$

and

$$
A_{j, N}:=\left(M_{1}^{j}, \ldots, M_{K_{j}}^{j}, M_{N}\right) \in \bar{A}(I) .
$$

Put $\quad h_{N}^{*, i}=F_{I_{i}}^{*}\left(A_{1, N}^{i}\right) \vee \cdots \vee F_{I_{i}}^{*}\left(A_{N, N}^{i}\right) \quad$ and $\quad h_{N}^{*}=F_{I}^{*}\left(A_{1, N}\right) \vee \cdots \vee F_{I}^{*}\left(A_{N, N}\right)$, $*=+,-$. We obtain $F_{I_{i}}^{*} \geq h_{N}^{*, i} \geq f_{N}^{*, i}$ and $F_{I}^{*} \geq h_{N}^{*} \geq f_{N}^{*}$. This implies $Z_{N}^{i}=$ $F_{I_{i}}-h_{N}^{+, i}+h_{N}^{-, i} \rightarrow G_{I_{i}}$ and $Z_{N}:=F_{I}-h_{N}^{+}+h_{N}^{-} \rightarrow G_{I}$, and (3.1.4) is proved.

We will call a process $F$ bounded, if

is finite.

$$
b(F):=\sup \left\{\Pi(I)^{-1} \int\left|F_{I}\right| d P: I \in I_{s}, \Pi(I)>0\right\}
$$

THEOREM (3.2). Let $F$ be a bounded and locally boundedly non-additive process. Set $J_{t}=[0, t \cdot e[, t \in \mathbb{Q}$. Then

$$
\lim _{t \rightarrow 0+} \prod\left(J_{t}\right)^{-1} F_{J_{t}}
$$

exists a.s.

Sketch of the proof. We can assume $\int F_{[0, r e[}^{\prime \prime} d P<+\infty$, for an $r>0$. Let $J=$ $\left[0, a\left[\subset\left[0, r \cdot e\left[\right.\right.\right.\right.$ with $a=\left(a_{i}\right)>0$. Let $b_{i}$ be the largest integer $n \leq r / a_{i}$ and let $c=\left(b_{i} \cdot a_{i}\right)$. We obtain

$$
\left(\prod_{i=1}^{l} b_{i}\right) \int F_{J}^{\prime \prime} d P \leq \int F_{[0, c[}^{\prime \prime} d P \leq \int F_{[0, r e[}^{\prime \prime} d P .
$$

This and $[0, r \cdot e[\subset[0,2 c[$ imply

$$
\prod(J)^{-1} \int F_{J}^{\prime \prime} d P \leq \prod\left(\left[0, c[)^{-1} \int F_{[0, r e[}^{\prime \prime} d P \leq 2^{l} \Pi\left(\left[0, r \cdot e[)^{-1} \int F_{[0, r e[}^{\prime \prime} d P\right.\right.\right.\right.
$$


So

$$
\begin{aligned}
& \sup \left\{\Pi(J)^{-1} \int F_{J}^{\prime \prime} d P: J \in I_{s}, \Pi(J)>0, J \subset[0, r \cdot e[\}\right. \\
& \quad \leq 2^{l} \Pi\left(\left[0, r \cdot e[)^{-1} \int F_{[0, r \mathrm{e}}^{\prime \prime} d P<+\infty\right.\right.
\end{aligned}
$$

is proved, and we obtain the decomposition (3.1.1) for all $I \subset[0, r \cdot e[$. By additivity and stationarity we extend $G$ to all $I \in I_{s}$. We obtain a bounded additive process, for which the existence of

$$
\lim _{\substack{t \rightarrow 0+\\ t \in \mathbf{O}}} \prod\left(J_{t}\right)^{-1} G_{J_{t}} \quad \text { a.s. }
$$

is proved in [1]. It remains to prove $\lim _{t \rightarrow 0+, t \in Q} \prod\left(J_{t}\right)^{-1} F_{J_{t}}^{*}=0$ a.s. for $*=+,-$. This can be done in almost the same manner as in the proofs of theorem (4.2), lemma (4.7) and lemma (4.8) in [1].

THEOREM (3.3). Let $F$ be a boundedly non-additive process with continuous parameter for which

$\sup \left\{\left|F_{[a, b]}\right|:[a, b[\subset[0, e[\right.$ and the coordinates of $a$ and $b$ are rational $\}$

is integrable. Set $J_{t}=[0, t \cdot e[, t \in \mathbb{Q}$. Then

$$
\lim _{\substack{t \rightarrow \infty \\ t \in \mathbf{Q}}} \prod\left(J_{t}\right)^{-1} F_{J_{t}}
$$

exists a.s.

The theorem can be proved by a reduction to a discrete case following the proof of theorem (2.5) in [1]. Both theorems remain valid if $\left\{J_{t}\right\}_{t \in Q}$ is a regular family of sets. One can define stationary processes indexed by more general sets than intervals, and it seems that the results carry over to that setting.

\section{Examples}

In this section we give some examples of non-additive processes, which appear in percolation on a lattice. These processes are given by a family of r.v.'s $F=\left(F_{I}, I \in I_{d}\right)$, where $\left(F_{I_{1}}, \ldots, F_{I_{n}}\right)$ has the same distribution as $\left(F_{I_{1}+u}, \ldots F_{I_{n}+u}\right)$, for all $I_{1}, \ldots, I_{n} \in$ $I_{d}, u \in \mathbb{N}_{0}^{d}$. As in the 1-parameter case (see [3]) we can pass to an equivalent process $\tilde{F}$ with sample space $\mathbb{R}^{I_{d}}$, which is a stationary process, defined as in definition (1.5).

Let the graph $L$ be given by a lattice of dimension $d \geq 2$, where the set $E$ of sites is $\mathbb{Z}^{d}$. Two points in $\mathbb{Z}^{d}$ are neighbours if their Euclidean distance is 1 . The bonds connect any two neighbours. The set of bonds will be denoted by $K$. All bonds are unoriented. Further let $\left\{U_{l}, l \in K\right\}$ be a set of non-negative i.i.d. r.v.'s with a finite mean see [5].

Example (4.1). We suppose $d=2$. For a fixed $0 \leq n \leq+\infty$ let $E_{n}:=\mathbb{Z} \times$ $\{k \in \mathbb{Z}:|k|<n+1\}$. Let the graph $L_{n}$ be given by the set of sites $E_{n}$ and the set of all bonds of $L$ whose end points are in $E_{n}$. For $l<n+1$ we define

$$
M_{m}^{l}:=\{(m, p):|p| \leq l\} \text {. }
$$

For a path $w$ consisting of a connected string $w_{1}, \ldots, w_{m}$ of bonds, put $U_{w}=\sum_{i=1}^{m} U_{w_{i}}$. Let $W_{m_{1}, m_{2}}^{l}$ be the set of all paths connecting $M_{m_{1}}^{l}$ to $M_{m_{2}}^{l}$. Put

$$
X_{m_{1}, m_{2}}^{l}(x):=\inf \left\{U_{w}(x): w \in W_{m_{1}, m_{2}}^{l}\right\}, \quad m_{1}<m_{2} .
$$


For $l=0, X=\left(X_{m_{1}, m_{2}}^{l}\right)$ is a subadditive process. For $l=n, X$ is a superadditive process. For $0<l<n, X_{0, m}^{\prime \prime \prime}$ is bounded by

$$
2 \sum_{j=0}^{m} \sum_{i=1-l}^{l} U_{((j, i-1),(j, i))}+\sum_{i=1}^{m} X_{i-1, i}^{l}-X_{0, m}^{l},
$$

$m \in \mathbb{N}$, so in this case $X$ is a boundedly nonadditive process.

Example (4.2). We suppose $d \geq 2$ and $\left\|U_{l}\right\|_{\infty}=: C<+\infty,(l \in K)$. For $I \in I_{d}$ let $L_{I}$ be the subgraph of $L$, whose set of sites is $E_{I}=\mathbb{Z}^{d} \cap I$, and whose set of bonds $K_{I}$ contains exactly all bonds of $L$ whose end points are in $E_{I}$. Let $S L_{I}$ be the set of all connected subgraphs of $L_{l}$, having the set of sites $E_{l}$, and which contain no circuit. We define pointwise:

$$
F_{I}(x):=\inf \left\{\sum_{l \in K^{\prime}} U_{l}(x): K^{\prime} \text { is the set of bonds of a graph } L^{\prime} \in S L_{l}\right\}, \quad I \in I_{d^{\prime}}
$$

We will now show that $F$ is a boundedly non-additive process. As $F$ is non-negative, this statement follows by (2.1.1) and (2.1.3) from: $F_{I}^{+}(x) \leq C \Pi(I)$, uniformly in $x$. To prove this fix $I \in I_{d}$ and $x \in X$. Let $A=\left(M_{i}\right)_{1 \leq i \leq m} \in \bar{A}(I)$. Suppose

$$
\sum_{\substack{J^{\prime} \subset J \\ J^{\prime} \in M_{i+1}}} F_{J}(x) \leq F_{J}(x)
$$

for a $j$ with $1 \leq j \leq m-1$ and a $J \in M_{j}$. Let, for $I^{\prime} \in I_{d}$ and $x \in X, L\left(I^{\prime}, x\right) \in S L_{I}$, be a graph with set of sites $E(I, x)$ and set of bonds $K\left(I^{\prime}, x\right)$, which satisfies

$$
F_{I}(x)=\sum_{l \in K\left(I^{\prime}: x\right)} U_{l}(x)
$$

We complete the graphs $L\left(J^{\prime}, x\right), J^{\prime} \in M_{j+1}, J^{\prime} \subset J$, to an element of $S L_{J}$. The set of bonds of this graph may be denoted by $K$. By the definition of $F$, we obtain

So we obtain:

$$
F_{J}(x) \leq \sum_{l \in K} U_{l}(x)
$$

$$
\left(F_{J}(x)-\sum_{\substack{J \subset J \\ J \in M_{j+1}}} F_{J}(x)\right)^{+} \leq C \cdot \operatorname{card}\left(K^{\prime} \backslash \bigcup_{\substack{J \subset J J \\ j \in M_{j+1}}} K\left(J^{\prime}, x\right)\right) .
$$

The number of new bonds we use for all completions like those described above is smaller than $\Pi(I)$ and they all are different; so $F_{I}^{+}(x) \leq C \Pi(I)$ is proved.

Acknowledgements. This paper contains a part of my dissertation written under the direction of Prof. U. Krengel; I want to thank Prof. U. Krengel for his guidance and encouragement.

\section{REFERENCES}

[1] M. A. Akcoglu \& U. Krengel. Ergodic theorems for superadditive processes. J. Reine Ang. Math. 323 (1981), 53-67.

[2] Y. Derriennic. Un theoreme ergodique presque sous-additif. Ann. Prob. 11 (1983), 669-677.

[3] A. del Junco. On the decomposition of a subadditive stochastic process. Ann. Prob. 5 (1981), 298-302. 
[4] J. F. C. Kingman. Subadditive processes. In Lecture Notes in Mathematics 539, 167-223. SpringerVerlag: Berlin-Heidelberg-New York, 1976.

[5] R. T. Smythe \& J. C. Wierman. First-Passage Percolation on the Square Lattice. Lecture Notes in Mathematics 671, Springer-Verlag: Berlin-Heidelberg-New York, 1978. 